\title{
An Architecture to Support QoS Multicast Routing for Ad-Hoc Networks
}

\author{
Beongku $\mathrm{An}^{1}$, Do Hyeon $\mathrm{Kim}^{2}$, and Nam-Soo Kim ${ }^{3}$ \\ ${ }^{1}$ School of Electrical, Electronic \& Computer Engineering, Hongik University \\ Jochiwon, Chungnam, Korea, 339-701,Tel.: +82-41-860-2243 \\ beongku@wow. hongik.ac.kr \\ ${ }^{2}$ School of Information and Communications, Cheonan University, Cheonan-City, \\ Chungnam, Korea, 330-180 Tel.: +82-41-620-9418, Fax: +82-41-620-9507 \\ dhkim@cheonan.ac.kr \\ ${ }^{3}$ Dept. of Computer and Communication Engineering, Chongju University, Chongju-City, \\ Chungbook, Korea, 360-764 Tel.: +82-43-229-8459 \\ nskim@chongju.ac.kr
}

\begin{abstract}
In this paper, we present an architecture for supporting QoS multicast routing in ad-hoc networks. The proposed architecture consists of three parts as follows. The first part is a clustering as underlying structure for supporting stable multicast services. In the second part, a framework which can support and evaluate the stability of route and network for supporting QoS routing is presented. In the third part, we describe a method which uses two structures of the first and second parts for supporting QoS multicast routing services.
\end{abstract}

\section{Introduction}

The goal of mobile ad-hoc wireless networking is to extend mobility into the realm of a set of wireless mobile nodes, where they form the network infrastructure in an adhoc fashion. Management functions, routing and multicasting in mobile ad-hoc wireless networks present more complex problems than in wired or last-hop networks, due to the random movement of nodes, the bandwidth and power limitations, and the lack of fixed infrastructure. In this paper, we present an architecture for supporting QoS multicast routing services for mobile ad-hoc wireless networks in those challenging environments as follows. First, a mobility-based clustering approaches and group based hierarchical structures are presented in order to facilitate the implementation of efficient and scalable multicasting techniques and mobility management functions. Second, we present a modeling framework to support and evaluate route and network stability for QoS routing services in mobile ad-hoc wireless networks. To support stable QoS routing services, the support and evaluation of the stability of route and network are very important. For example, if we can select most stable route among multiple routes between source node and destination node, we can save much bandwidth and reduce delay and overload. Third, we describe a method which can support QoS multicast routing services using two structures presented above.

The remaining of this paper is organized as follows. Section 2 describes the architecture for supporting QoS multicast routing services in mobile ad-hoc wireless net 
works. Section 3 contains the performance evaluation of our proposed methods, while section 4 concludes the paper.

\section{The Architecture}

First, we introduce a mobility-based clustering structure to support a stable multicast service. The main idea of the clustering is to combine both physical and logical partitions of the network as well as the concept of relative mobility in order to improve the stability in the clustered topology. The proposed mobility-based hierarchical clustering algorithm[1] may result in variable-size clusters depending on the mobility characteristics of the nodes. A group may consist of clusters that present similar mobility characteristics. Several groups can be hierarchically merged into one group depending on the mobility of each group. Fig. 1 shows the basic concepts of the proposed clustering.

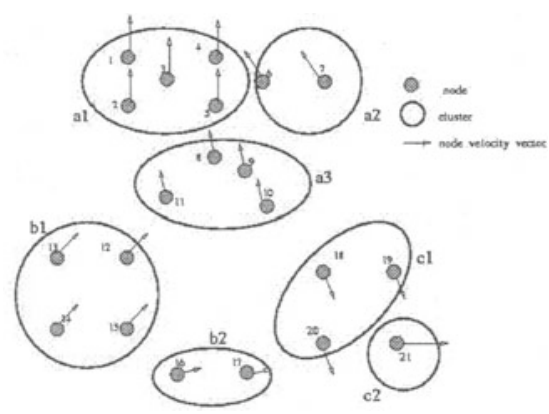

Fig. 1. Basic concepts of the clustering

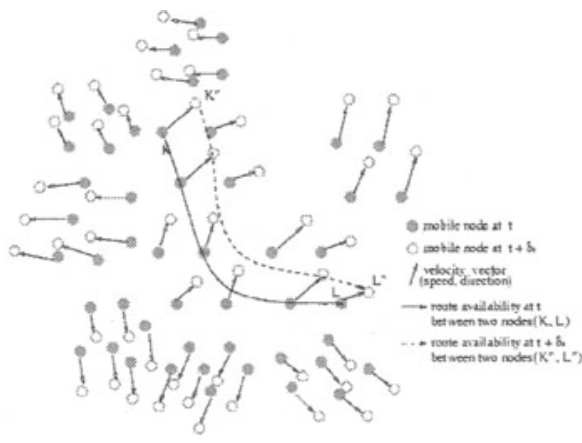

Fig. 2. Route stability for supporting QoS routing

Second, we present a modeling framework for supporting QoS routing. The main goal of the presented modeling framework is to select the most stable route between a source and a destination, in an environment where multiple paths are available, as well as to create a convenient performance measure to be used for the evaluation of the stability and connectivity in a mobile ad-hoc wireless networks. The basic motivations of the proposed modeling approach stem from the commonality observed in the location uncertainty in mobile ad-hoc wireless networks and the concept of entropy [2]. We also associate each node $\mathrm{m}$ with a set of variable features denoted by $a_{m, n}$ where node $\mathrm{n}$ is a neighbor of node $\mathrm{m}$. In this paper, two nodes are considered neighbors if they can reach each other in one hop (e.g. direct communication). These variable features $a_{m, n}$ represent a measure of the relative speed among two nodes and are defined rigorously later in this section. Any change of the system can be described as a change of variable values $a_{m, n}$ in the course of time $\mathrm{t}$ such as $a_{m, n}(t) \rightarrow a_{m, n}\left(t+\Delta_{t}\right)$. Let us also denote by $\mathrm{v}(\mathrm{m}, \mathrm{t})$ the velocity vector of node $\mathrm{m}$ and by $v(n, t)$ the velocity vector of node $n$ at time $t$. Please note that velocity vectors $v(m, t)$ and $v(n, t)$ have two parameters, namely speed and direction. The relative ve- 
locity $\mathrm{v}(\mathrm{m}, \mathrm{n}, \mathrm{t})$ between nodes $\mathrm{m}$ and $\mathrm{n}$ at time $\mathrm{t}$ is defined as: $v(m, n, t)=v(m, t)-v(n, t)$. Then, the relative mobility between any pair $(\mathrm{m}, \mathrm{n})$ of nodes during some time interval is defined as their absolute relative speed averaged over time. Therefore, we have:

$a_{m, n}=\frac{1}{N} \sum_{i=1}^{N}\left|v\left(m, n, t_{i}\right)\right|$

. where $N$ is the number of discrete times $t_{i}$ that velocity information can be calculated and disseminated to other neighboring nodes within time interval. Based on this, we can define the entropy $H_{m}\left(t, \Delta_{t}\right)$ at mobile $m$ during time interval. The entropy can be defined either within the whole neighboring range of node $m$, or for any subset of neighboring nodes of interest. In general, the entropy $H_{m}\left(t, \Delta_{t}\right)$ at mobile $m$ is calculated as follows:

$H_{m}\left(t, \Delta_{t}\right)=\frac{-\sum_{k \in F_{m}} P_{k}\left(t, \Delta_{t}\right) \log P_{k}\left(t, \Delta_{t}\right)}{\log C\left(F_{m}\right)}$, where $P_{k}\left(t, \Delta_{t}\right)=\frac{a_{m, k}}{\sum_{i \in F_{m}} a_{m, i}}$. In this relation by $F_{m}$, we denote the set (or any subset) of the neighboring nodes of node $m$, and by $C\left(F_{m}\right)$ the cardinality (degree) of set $F_{m}$. If we want to calculate the local network stability (with reference to node $m$ ), then $F_{m}$ refers to the set that includes all the neighboring nodes of mobile node $m$, while if we are interested in the stability of a part of a specific route then $F_{m}$ represents the two neighboring nodes of mobile node m over that route. As can be observed from the previous relation, the entropy $H_{m}\left(t, \Delta_{t}\right)$ is normalized so that $0 H_{m}\left(t, \Delta_{t}\right) \quad 1$. It should be noted that the entropy, as defined here, is small when the change of the variable values in the given region is severe and large when the change of the values is small [2]. Let us present the route stability (RS) between two nodes $k$ and $l$ during some interval $\Delta_{t}$ as $\gamma=R S_{k, l}\left(t, \Delta_{t}\right)$. We also define and evaluate two different measures to estimate and quantify end to end route stability, denoted by $\gamma^{1}=R S_{k, l}^{1}\left(t, \Delta_{t}\right)$ and $\gamma^{2}=R S^{2}{ }_{k, l}\left(t, \Delta_{t}\right)$ and defined as follows respectively:

$$
\begin{gathered}
\gamma^{1}=R S_{k, l}^{1}\left(t, \Delta_{t}\right)=\prod_{t=1}^{N_{r}}\left[H_{i}\left(t, \Delta_{t}\right)\right] \\
\gamma^{2}=R S_{k, l}^{2}\left(t, \Delta_{t}\right)=\min _{i=\left[1,2,3, \ldots, N_{r}\right]}\left[H_{i}\left(t, \Delta_{t}\right)\right]
\end{gathered}
$$

where $\mathrm{Nr}$ denotes the number of intermediate mobile nodes over a route between the two end nodes $(\mathrm{k}, \mathrm{l})$. Parameter $\gamma^{1}\left(\gamma^{2}\right)$ can be used to measure the route availability and stability.

Third, we present a method which uses two structures of the first and second parts for supporting QoS multicast routing services. The main objective for supporting QoS multicast routing is to reduce the unnecessary routes and the redundancy for bandwidth saving as well as delay and overload. Fig. 3 describes the steps for supporting QoS multicast routing on an example multicast routing protocol, namely MHMR[3], for mobile ad-hoc wireless networks. In first step (Fig.3(a)), MHMR uses a limited 
mesh structure over the clusterheads only that are created by the underlying mobilitybased clustering. In second step (Fig.3(b)), although the MHMR creates a mesh infrastructure for multicast routing, we are trying to use a source-based tree for multicast packet forwarding to support QoS multicast routing. Then, we can send multicast message data over the most stable single route among the mesh routes depending on the stability of routes. Therefore, we can support QoS multicast routing services(i.e., reduce redundancy and unnecessary routes for bandwidth saving, reduce delay and overload, increase throughput) in mobile ad-hoc wireless networks.

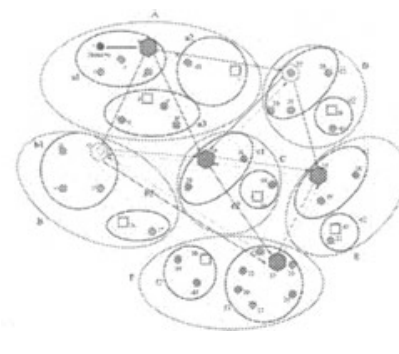

(a) Mesh structure
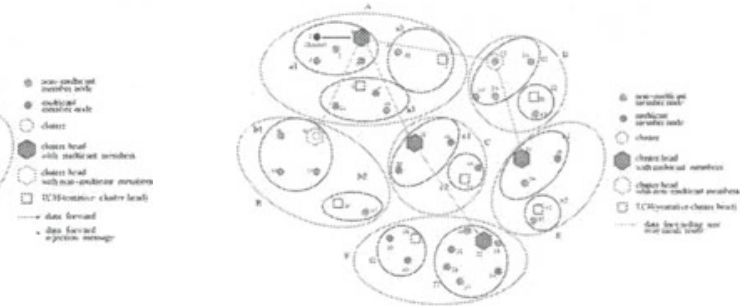

Fig. 3. Supporting QoS multicast routing

\section{Evaluation and Discussion}

The performance evaluation of our protocol is accomplished via modeling and simulation using the Optimized Network Engineering Tool (OPNET). A mobile ad-hoc network consisting of 200 nodes that are placed randomly within a rectangular region of $2 \mathrm{~km} \mathrm{x} 2 \mathrm{~km}$ is modeled in the simulation. Each node is modeled as an infinitebuffer, store-and-forward queuing station, and is assumed to be aware of its position with the aid of a reliable position location system (i.e., GPS). The mobile nodes are assumed to have constant radio range of $\mathrm{Z}=250 \mathrm{~m}$. Two different mobility scenarios are considered in this study. In the first one (in the following, we refer to it as mobility 1), the speed and the direction of each move are uniformly distributed with speed range $[0, V \max \mathrm{km} / \mathrm{h}]$ and direction range $[0,2 \pi]$ respectively. In the second one (in the following, we refer to it as mobility 2), a group-based mobility pattern[4] is modeled. Specifically, nodes are grouped into several groups, where we assume that nodes in the same group have similar mobility characteristics (speed and direction). The speed and direction of each group are selected randomly at the start point of the simulation within the speed range $[0, \mathrm{Vmax} \mathrm{km} / \mathrm{h}]$ and the direction range $[0,2 \pi]$ and is assumed that the group holds these speed and direction for the duration of the simulation. Initially, each group consists of 10 nodes. If a mobile arrives at the boundary of the given network coverage area, the node reenters into network.

In the first part simulation, we measure and evaluate the stability of the created clustered topology for supporting multicast. The used metrics is number of cluster changes that represent a measure of the rate of clusterhead changes (or construction of 
new clusters). Fig. 4 presents the cluster stability for individual random mobility scenario (i.e., mobility 1) under three different clustering algorithms: lowest-ID algorithm[5], highest-connectivity (degree) algorithm[6], and MBC algorithm. Similarly, Figure 5 shows the corresponding cluster stability under the group mobility scenario (i.e. mobility 2). As can be seen by the first set of fig. 4, MBC algorithm outperforms both of them by improving the cluster stability metrics. The improvement is considerably higher in the experiment using group mobility scenario (Fig. 5) where MBC algorithm reduces the clusterhead change ratio by approximately several times compared to the lowest-ID algorithm and the highest-connectivity algorithm. This is achieved because MBC algorithm takes advantage of the similarity that several nodes demonstrate in this set of experiment due to the group mobility pattern assumed.

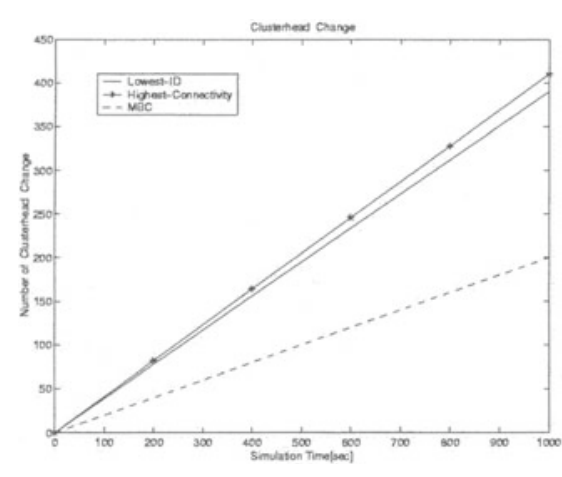

Fig. 4. Cluster Stability-Mobility 1

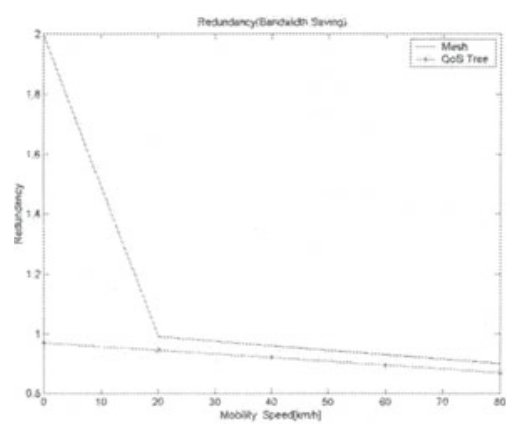

Fig. 6. Redundancy(Bandwidth Saving)Mobility2

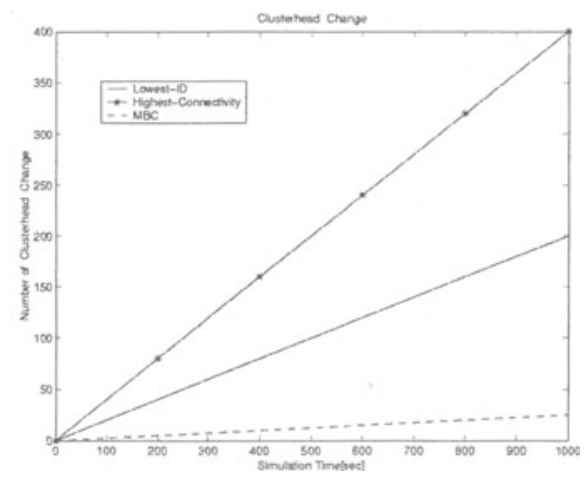

Fig. 5. Cluster Stability-Mobility 2

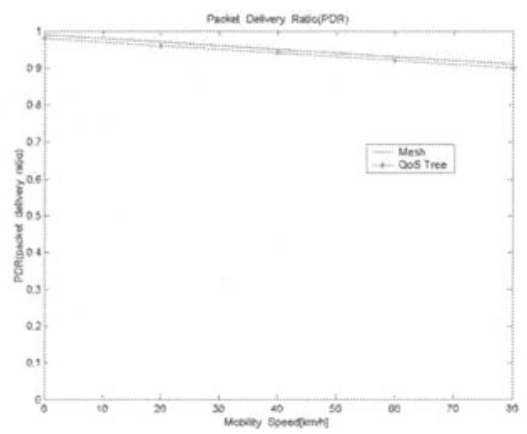

Fig. 7. Packet delivery ratio-Mobility2

In the next part simulation, we are trying to measure and evaluate the degree of QoS multicast routing on the implemented multicast routing protocol, namely MHMR [3]. The performance metrics, redundancy \& bandwidth saving, are studied. Fig. 6 present the degree of redundancy for group mobility at one destination node per one packet sending from one source as mobility function, while fig. 7 present the packet delivery ratio as mobility function group mobility. As can be seen by figure 6 and fig. 
7, even though the redundancy using QoS tree is less than the redundancy using just mesh concept, as can be seen by figure 6 , the packet delivery ratio is similar for mesh by fig. 7. The reason is that QoS tree can select the most stable single route among the multiple routes between source node and destination node.

\section{Conclusions}

In this paper, we present an architecture for supporting QoS multicast routing in mobile ad-hoc wireless networks. The proposed architecture consists of three parts as follows. First, we present a clustering structure which can support stable multicast routing services. Second, a framework which can support and evaluate the stability of route and network for supporting QoS routing is presented. Third, we present a method for supporting QoS multicast routing services in mobile ad-hoc wireless networks. The performance evaluation of our proposed methods is accomplished via modeling and simulation. The performance results demonstrate that our proposed architecture can efficiently support QoS multicast routing services in mobile ad-hoc wireless networks.

\section{References}

1. R. Ramanathan and M. Steenstrup, "Hierarchically-Oragnized, Multihop Mobile Wireless Networks for Quality-of-Service Support", ACM/Baltzer Mobile Networks and Applications, vol. 3, no. 1, p.p. 101-119, 1998.

2. Akira Shiozaki, "Edge Extraction Using Entropy Operator", Computer Vision, Graphics, and Image Processing 36, 1-9, 1986.

3. Beongku An and Symeon Papavassiliou, "MHMR: A Mobility-Baed Hybrid Multicast Routing Protocol in Mobile Ad-hoc Wireless Networks", Wireless Communications and Mobile Computing (WCMC), vol.3, issue 2, pp.255-270, 2003.

4. Xiaoyan Hong, Mario Gerla, Guangyu Pei and Ching-Chuan Chiang, "A Group Mobility Model for Ad-Hoc Wireless networks”, Proc. of ACM/IEEE MSWiM'99, August 1999.

5. Anthony Ephremides, Jeffrey E. Wieselthier, and Dennis J. Baker, "A design concept for reliable mobile radio networks with frequency hopping signaling", Proc. of IEEE 75(1), pp.56-73, 1987.

6. Abhay K. Parekh, "Selecting routers in ad-hoc wireless networks", in ITS, 1994. 